\title{
Human Umbilical Cord Mesenchymal Stem Cells Attenuate Abdominal Aortic Aneurysm Progression in Sprague-Dawley Rats: Implication of Vascular Smooth Muscle Cell Phenotypic Modulation
}

\author{
Hao Wen, ${ }^{1-3}$ Mingjing Wang, ${ }^{4}$ Shiqiang Gong, ${ }^{4}$ Xintong Li, ${ }^{1-3}$ Jinze Meng, ${ }^{4}$ \\ Jie Wen, ${ }^{5}$ Yifei Wang, ${ }^{4}$ Shuqing Zhang, ${ }^{4}$ and Shijie $\mathrm{Xin}^{1-3}$
}

\begin{abstract}
Abdominal aortic aneurysm (AAA) is life-threatening, for which efficient nonsurgical treatment strategy has not been available so far. Several previous studies investigating the therapeutic effect of mesenchymal stem cells (MSCs) in AAA indicated that MSCs could inhibit aneurysmal inflammatory responses and extracellular matrix destruction, and suppress aneurysm occurrence and expansion. Vascular smooth muscle cell (VSMC) phenotypic plasticity is reported to be predisposed in AAA initiation and progression. However, little is known about the effect of MSCs on VSMC phenotypic modulation in AAA. In this study, we investigate the therapeutic efficacy of umbilical cord mesenchymal stem cells (UC-MSCs) in elastase-induced AAA model and evaluate the effect of UC-MSC on VSMC phenotypic regulation. We demonstrate that the intravenous injection of UC-MSC attenuates elastase-induced aneurysmal expansion, reduces elastin degradation and fragmentation, inhibits MMPs and TNF$\alpha$ expression, and preserves and/or restores VSMC contractile phenotype in AAA. Taken together, these results highlight the therapeutic and VSMC phenotypic modulation effects of UC-MSC in AAA progression, which further indicates the potential of applying UC-MSC as an alternative treatment candidate for AAA.
\end{abstract}

Keywords: umbilical cord mesenchymal stem cells, abdominal aortic aneurysm, vascular smooth muscle cells, contractile phenotype, cellular therapy

\section{Introduction}

A BDOMINAL AORTIC ANEURYSM (AAA) is estimated to account for 150,000-200,000 deaths every year and causes serious socioeconomic burden worldwide [1]. Open surgical repair (OSR) with artificial blood vessel is effective to prevent aneurysm rupture, but is associated with high perioperative morbidity and mortality rate [2]. Endovascular aneurysm repair (EVAR) is less invasive and generates lower perioperative comorbidity rate than OSR in short-term duration; however, benefits of EVAR are not sustained due to higher reintervention rate and secondary rupture caused by endoleaks or endograft durability [3-5]. Elegant studies have been conducted aiming to explore candidate drugs for stabilization of aneurysm growth, yet no medical therapy can be recommended [6]. Hence, new noninvasive alternative strategies are truly needed to halt or reverse aneurysm expansion.

The typical pathological characteristics of AAA are extracellular matrix (ECM) degradation, medial vascular smooth muscle cell (VSMC) dysfunction and loss, and inflammatory cell and cytokine infiltration [7]. VSMCs are the most predominant cell type within the aortic wall; the abnormality and dysfunction of VSMCs could impair vascular homeostasis and structural integrity [8], which further contributes to the occurrence and progression of AAA [9-11]. Under normal physiological conditions, VSMCs preserve a contractile phenotype, which is featured by high expression of contractile proteins essential for contraction machinery and cell anchorage, such as

\footnotetext{
${ }^{1}$ Department of Vascular Surgery, The First Affiliated Hospital of China Medical University, Shenyang, China.

${ }^{2}$ Key Laboratory of Pathogenesis, Prevention and Therapeutics of Aortic Aneurysm in Liaoning Province, Shenyang, China.

${ }^{3}$ Regenerative Medicine Research Center of China Medical University, Shenyang, China.

${ }^{4}$ Department of Pharmacology, China Medical University, Shenyang, China.

${ }^{5}$ Department of Ultrasonography, Inner Mongolia Baotou City Central Hospital, Baotou, China.
}

(C) Hao Wen et al. 2020; Published by Mary Ann Liebert, Inc. This Open Access article is distributed under the terms of the Creative Commons Attribution Noncommercial License (http://creativecommons.org/licenses/by-nc/4.0/) which permits any noncommercial use, distribution, and reproduction in any medium, provided the original author(s) and the source are cited. 
SMA- $\alpha$ and SM-22 [8,12]. While in pathological stimulation, VSMCs undergo dedifferentiation and exhibit a synthetic phenotype, which is characterized by enhanced proliferation and migration abilities and high expression of synthetic proteins like OPN $[8,13]$.

Recently, preclinical studies have found mesenchymal stem cell (MSC)-based cellular therapy is beneficial to AAA initiation and progression [14-21]. Parvizi et al. [20,21] demonstrated that perivascular scaffolds loaded with adipose-derived MSCs could attenuate AAA development, prevent loss of VSMCs, and decrease macrophage infiltration in rats. Sharma et al. [19] found that intravenous injection of umbilical cord mesenchymal stem cells (UC-MSCs) attenuates proinflammatory cytokine IL-17 production and protects against AAA formation. However, these investigations mainly focused on the ability of MSCs to modulate inflammatory and immune responses in preventing AAA occurrence and development. Little is known about the VSMC phenotypic modulation in stem cell-based therapy for AAA.

In this study, we examined the therapeutic effects and efficacy of UC-MSCs in the management of AAA, and highlight the preservation and/or restoration roles of UCMSC treatment on VSMC phenotypic transition.

\section{Materials and Methods}

\section{Acquisition of human UC tissue and culture of UC-MSCS}

The study protocols were approved by the Ethics Committee of the First Affiliated Hospital of China Medical University, in accordance with the Declaration of Helsinki. Tissue donors gave written informed consent before the study. The primary culture protocols of UC-MSCs were followed as previously described [22]. The fresh umbilical cord tissue was extracted aseptically, and was cut open longitudinally; one umbilical vein and two umbilical arteries were removed to expose Wharton jelly tissue. Then, the residual blood was washed repeatedly with normal saline, and the remaining Wharton jelly was cut into small pieces of $1 \mathrm{~mm}^{3}$ with ophthalmic scissors and transferred into a T75 cell culture flask. Dulbecco's modified Eagle's medium/Ham's F12 medium (11320033; Gibco) containing 10\% fetal bovine serum (FND500; ExCell Bio) and 1\% penicillin/streptomycin (15070063; Gibco) was added, and cells were cultured in a $5 \% \mathrm{CO}_{2}$-/water-saturated incubator at $37^{\circ} \mathrm{C}$. The status of cell growth was observed under inverted microscope (Eclipse TS100; Nikon) every day. The culture medium was changed for the first time after 1 week, and then changed every 2 days. When the cells reached $80 \%-90 \%$ confluence, cells were digested and passaged with $0.25 \%$ trypsin-EDTA (25200072; Gibco), and the digestion time was controlled under the microscope. Cells between passage 3 (P3) and passage 5 (P5) were used in this study.

\section{Cell proliferation assay}

The cell proliferation analysis was conducted by using Cell Counting Kit-8 (CCK-8) assay (CK04; Dojindo). Briefly, cells were plated in 96-well plates at a density of $2 \times 10^{3}$ cells/well in $100 \mu \mathrm{L}$ culture medium, the absorbance was continuously measured at the same time point from 1 to 7 days after cell implantation. According to the manufacturer's protocol, $10 \mu \mathrm{L}$ of CCK-8 solution was added to each well, and the plate was incubated for $4 \mathrm{~h}$ before the absorbance was measured at
$450 \mathrm{~nm}$ on a microplate reader (iMark ${ }^{\mathrm{TM}}$ Microplate Absorbance Reader; Bio-Rad Laboratories).

\section{Cell cycle analysis}

Cell cycle analysis was carried out by flow cytometry technique to check the percentages of cells in different cell cycle stages. Briefly, cells were seeded at a density of $5 \times 10^{5}$ cells/ dish into a $10 \mathrm{~cm}^{2}$ cell culture dish; after incubating at $37^{\circ} \mathrm{C}$ for $24 \mathrm{~h}$, cells were washed thrice with phosphate-buffered saline (PBS) and fixed with $75 \%$ precooled ethanol for $12 \mathrm{~h}$. After washing with PBS three times, the fixed cells were resuspended with $0.5 \mathrm{~mL}$ propidium iodide (PI) staining working solution (C1052-2; Beyotime) containing 2\% RNase A (C1052-3; Beyotime) and incubated in dark at $37^{\circ} \mathrm{C}$ for $30 \mathrm{~min}$. Then, the cells were consigned to a MACSQuant ${ }^{\circledR}$ Analyzer Flow Cytometer (Miltenyi Biotec) for further analysis.

\section{Induction of adipogenic, osteogenic, and chondrogenic differentiation of UC-MSCs}

For adipogenic differentiation, UC-MSCs were seeded at a density of $5 \times 10^{4}$ cells/well into a six-well plate and incubated with $2 \mathrm{~mL} /$ well MesenCult ${ }^{\mathrm{TM}}$ Adipogenic Differentiation Medium (05412; Stem Cell Technologies) in a 5\% $\mathrm{CO}_{2}$-/water-saturated incubator at $37^{\circ} \mathrm{C}$ for 25 days; the induction medium was changed every 3 days. Finally, the cells were fixed with $4 \%$ paraformaldehyde and the intracellular lipid accumulation of UC-MSCs was visualized by oil red $\mathrm{O}$ staining as previously described [23].

For osteogenic differentiation, UC-MSCs were seeded at a density of $5 \times 10^{4}$ cells/well into a six-well plate and incubated with $2 \mathrm{~mL} /$ well MesenCult Osteogenic Differentiation Medium (05465; Stem Cell Technologies) in a $5 \% \mathrm{CO}_{2}$-/watersaturated incubator at $37^{\circ} \mathrm{C}$ for 15 days; the induction medium was changed every 3 days. Finally, the cells were fixed with $4 \%$ paraformaldehyde and the intracellular calcium accumulation of UC-MSCs was visualized by Alizarin Red S staining as previously described [23].

For chondrogenic differentiation, a total of $5 \times 10^{5}$ UCMSCs were suspended and centrifuged into a $15 \mathrm{~mL}$ polypropylene tube as a cell pellet and incubated with $0.5 \mathrm{~mL}$ MesenCult-ACF (animal component-free) Chondrogenic Differentiation Medium (05455; Stem Cell Technologies) in a $5 \% \mathrm{CO}_{2}$-/water-saturated incubator at $37^{\circ} \mathrm{C}$ for 3 days first; then, $0.5 \mathrm{~mL}$ of complete induction medium was added on day 3 and was replaced with $0.5 \mathrm{~mL}$ medium after every 3 days until day 21. Finally, the chondrogenic pellet was fixed with $10 \%$ formalin, followed by subsequent standard paraffin embedding and visualized by hematoxylin-eosin (H\&E) staining.

\section{Cell surface markers analysis}

Flow cytometry was implemented for measuring UC-MSC surface markers. Briefly, cells were incubated with FITCconjugated primary antibodies after preprocessing steps as described in the section of cell cycle analysis. Then, the cells were subjected to a MACSQuant Analyzer Flow Cytometer (Miltenyi Biotec) for analysis. The primary antibodies used in this experiment are mouse anti-human CD34 (555821; BD Pharmingen), mouse anti-human CD45 (555482; BD Pharmingen), mouse anti-human CD73 (561254; BD Pharmingen), mouse anti-human CD90 (561969; BD Pharmingen), mouse anti- 
human CD105 (561443; BD Pharmingen), and mouse antihuman HLA-DR (555560; BD Pharmingen).

\section{Animals}

All animals were cared for according to the Guide for the Care and Use of Laboratory Animals stated by the U.S. National Institutes of Health. All the experimental procedures were approved by the Animal Research Ethics Committee of China Medical University. A total of 30 male 8-10 weeks old Sprague-Dawley (SD) rats (Changsheng Biotechnology) were used in this study.

\section{Establishment of $A A A$ model and experimental design}

In this study, elastase infusion method was implemented to establish an in vivo model of AAA in male SD rats following previous established protocols [24]. Briefly, after animals were anesthetized with gas mixture of $2 \%$ isoflurane and $2 \mathrm{~L} / \mathrm{min}$ oxygen, midline abdominal incision was performed, the segment between left renal vein and bifurcation of abdominal aorta was carefully isolated and exposed, and then the infrarenal abdominal aorta was perfused with elastase $(30 \mathrm{U} / \mathrm{mL}$; Solarbio Life Sciences) or saline through PE-10 catheter under $130 \mathrm{~cm}$ water column pressure for $30 \mathrm{~min}$.

All rats were randomly divided into three groups: the control group $(n=10)$ was perfused with saline and injected with $1 \mathrm{~mL}$ saline through tail vein on day 1 (the first postoperative day); the elastase group $(n=10)$ was perfused with active elastase and injected with $1 \mathrm{~mL}$ saline through tail vein on day 1 ; and the elastase+MSC group was perfused with active elastase and injected with $1 \times 10^{6}$ UC-MSCs in $1 \mathrm{~mL}$ saline through tail vein on day 1 (Fig. 2A).

The diameter and morphology of abdominal aorta was evaluated by ultrasound, $\mathrm{H} \& \mathrm{E}$ staining, and elastic fiber staining. Immunohistochemistry (IHC) staining, immunofluorescence staining, and western blot analysis were used to evaluate the expression level of VSMC contractile phenotypic markers among the three groups. Throughout this study, the experimenters were all blind to the analysis of data and the sample size for each experiment was illustrated in the corresponding figure legend.

\section{Measurements of abdominal aorta diameter by ultrasound}

All animals were anesthetized with isoflurane inhalation after abdominal depilation. The maximum internal diameter of infrarenal abdominal aorta of each animal was measured by ultrasound cross-sectionally before aortic perfusion (day 0 ) and on the 7th and 14th day after operation. Vevo 770 system (VisualSonics) was used for ultrasonic measurements according to the manufacturer's instructions.

\section{Histological analysis}

Abdominal aorta samples were fixed in $4 \%$ paraformaldehyde for $24 \mathrm{~h}$, embedded in paraffin, and sectioned into $4 \mu \mathrm{m}$ thickness. After standard deparaffinization and rehydration steps, sections were H\&E stained to assess morphological changes. Moreover, Gomori aldehyde fuchsin staining method (G1593; Solarbio Life Sciences) was implemented to evaluate the changes of elastic fibers within the arterial wall. According to the manufacturer's protocols, after deparaffinization and rehydration, sections were incubated with acid oxidation solution for $5 \mathrm{~min}$, washed with PBS, and then incubated with acid bleaching solution for $5 \mathrm{~min}$. After washing with PBS and $70 \%$ ethanol, sections were incubated with aldehyde fuchsin for $10 \mathrm{~min}$ and orange $\mathrm{G}$ for $1-2 \mathrm{~s}$.

IHC staining and immunofluorescence staining were performed to evaluate the expression level of VSMC contractile phenotypic markers SMA- $\alpha$ and SM-22 within arterial walls. For IHC analysis, sections were deparaffinized and rehydrated, and then incubated with $3 \% \mathrm{H}_{2} \mathrm{O}_{2}$ to deactivate endogenous peroxidase at room temperature in dark for $10 \mathrm{~min}$, followed by heat-induced antigen retrieval with pressure cooker containing sodium citrate buffer $(10 \mathrm{mM}, \mathrm{pH} 6.0)$ and blocking with normal goat serum for $30 \mathrm{~min}$ at room temperature. After that, sections were incubated with primary antibodies at $4^{\circ} \mathrm{C}$ in a humidified chamber overnight and HRP-conjugated goat anti-rabbit secondary antibody (1:2,000, ab205718; Abcam) at room temperature for $1 \mathrm{~h}$. Sections were detected with diaminobenzidine (DAB) and stained with hematoxylin before dehydration and examination under microscopy.

For immunofluorescence analysis, sections were deparaffinized and rehydrated as abovementioned, followed by heatinduced antigen retrieval, permeabilization with Triton X-100, and blocking with $5 \%$ bovine serum albumin (BSA) for $30 \mathrm{~min}$ at room temperature. After that, sections were incubated in dark with primary antibodies at $4^{\circ} \mathrm{C}$ in a humidified chamber overnight with Alexa Fluor 488 (1:200, ab150073; Abcam)- or Alexa Fluor 647 (1:200, ab150075; Abcam)-conjugated donkey anti-rabbit secondary antibody at room temperature for $1 \mathrm{~h}$. Nuclei were stained with DAPI before visualization with confocal microscope (C2+ system; Nikon). Images were analyzed and quantified with ImageJ software (version 1.52t; $\mathrm{NIH}$ ). For elastic fiber quantification, the mean areas of elastic fibers were measured following ImageJ user's guide and the number of elastin breaks was manual counted per section.

For immunofluorescence staining, the average fluorescence intensities of SMA- $\alpha$, SM-22, and OPN in the medial wall area were measured per section according to the ImageJ user's guide. For IHC staining, the IHC Toolbox plugin (http://imagej.nih.gov/ij/plugins/ihc-toolbox) in ImageJ was applied to measure the average intensities of positive signals per section. All data were exported to Prism software to generate plots. The primary antibodies used in IHC and immunofluorescence experiments were anti-SMA- $\alpha(1: 100$, ab5694; Abcam) and anti-SM-22 (1:100, ab155272; Abcam).

\section{Western blot analysis}

Total protein was extracted from fresh aorta samples using RIPA lysis buffer (P0013B; Beyotime) containing 2\% protease and phosphatase inhibitor cocktail (P1050; Beyotime). The concentration of total protein was determined by a BCA protein quantitative kit (P0012S; Beyotime). A total of $40 \mu \mathrm{g}$ protein per sample was separated by $10 \%$ sodium dodecyl sulfate-polyacrylamide gel electrophoresis (P0012A; Beyotime) and then electrophoretically transferred onto a $0.2 \mu \mathrm{m}$ polyvinylidene difluoride (PVDF) membrane (88520; Thermo Fisher Scientific) using Trans-Blot ${ }^{\circledR}$ SD Semi-Dry Transfer Cell System (Bio-Rad Laboratories). The membranes were blocked with $1 \%$ BSA for $2 \mathrm{~h}$ at room temperature and incubated with primary antibodies for $12 \mathrm{~h}$ at $4^{\circ} \mathrm{C}$. Then, after washing thrice 
(10 min each), membranes were further incubated with HRPconjugated secondary antibodies (1:3,000, ab205718; Abcam) for $1 \mathrm{~h}$ at room temperature. Chemiluminescence visualization was conducted using Hypersensitive ECL Luminescent Solution (SB-WB002L; Share-Bio Technology, Shanghai, China). Intensities of protein bands were measured with ImageJ software (Version 1.52t; NIH), and GAPDH was used as internal reference protein.

The following primary antibodies were used in this experiment: anti-SMA- $\alpha$ (1:3,000, ab5694; Abcam), anti-SM-22 (1:3,000, ab155272; Abcam), anti-OPN (1:1,000, BM4208; Boster Biological Technology), anti-MMP-2 (1:5,000, NB200193; Novus Biological), anti-MMP-9 (1:2,000, AF5228; Affbiotech), anti TNF- $\alpha$ (1:1,000, PB0082; Boster Biological Technology), and anti-GAPDH (1:2,000, BM3896; Boster Biological Technology).

\section{Statistical analysis}

In this study, results are expressed as mean \pm standard error of the mean and statistical significance between the means of two groups was determined by two-tailed unpaired $t$-test or Mann-Whitney test. $P$ value of $<0.05$ was considered statistically significant. GraphPad Prism software (version 8.2.1; GraphPad Prism Software, Inc., San Diego, CA) was used for all statistical analyses.

\section{Results}

\section{Isolation and characterization of UC-MSCS}

In the processes of expansion and culture in vitro, P3 and P5 UC-MSCs were well adhered to the plastic surface and exhibited homogenous fibroblast-like morphology and
A

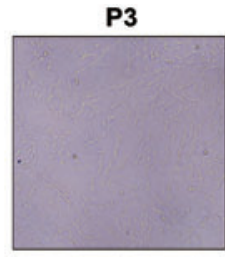

C

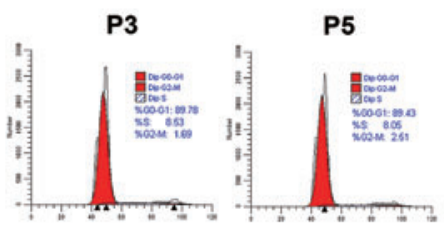

E
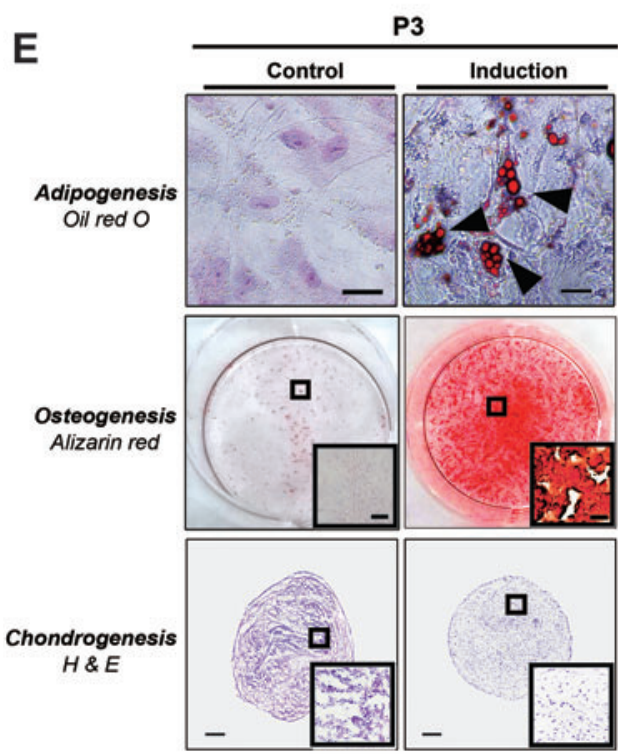

B

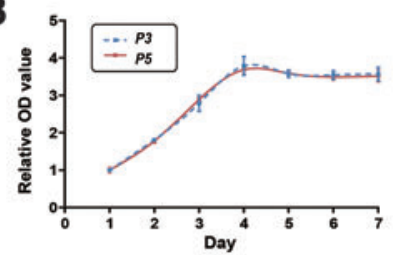

D
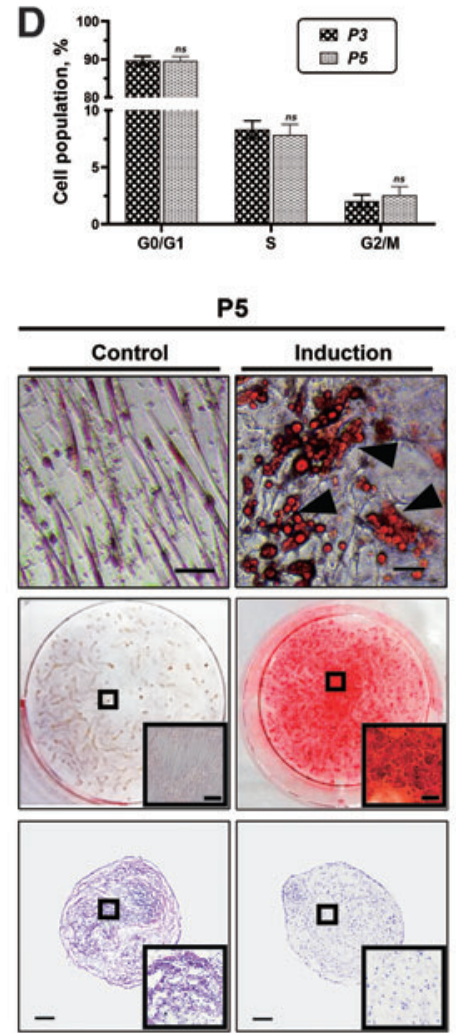

F
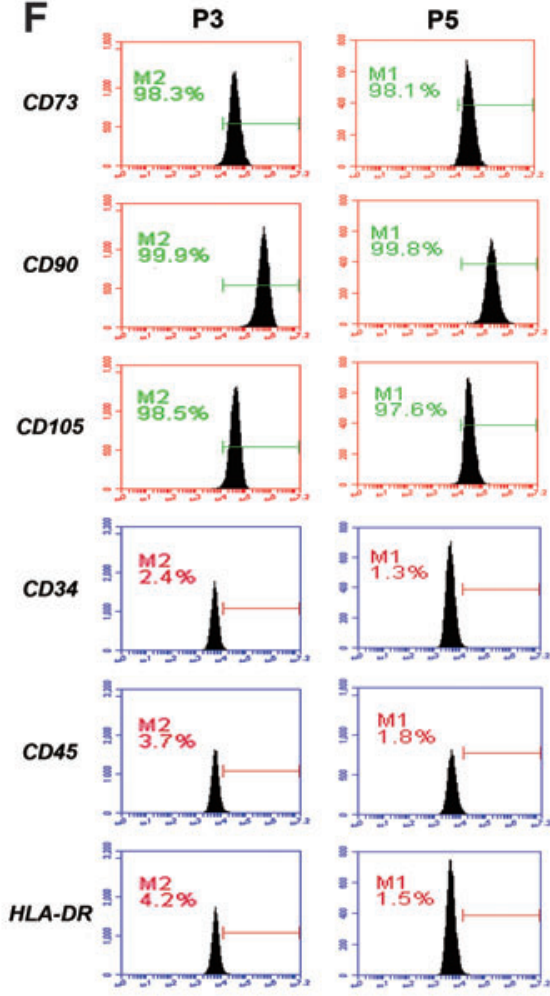

G

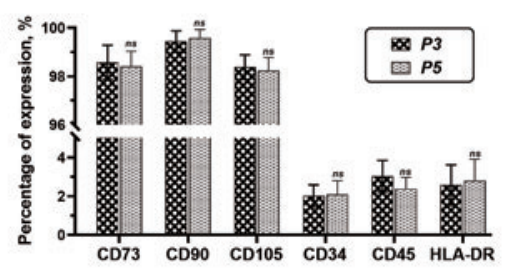

FIG. 1. Characterization of human UC-MSCs. (A) Fibroblast-shaped morphology of passage 3 (left) and passage 5 (right) UC-MSCs under inverted microscope ( $\times 100$ magnification). (B) Cell proliferation analyses of passage 3 (blue) and passage 5 (red) UC-MSCs; the data indicate mean \pm SEM of five independent experiments. (C) Cell cycle analyses of passage 3 (left) and passage 5 (right) UC-MSCs. (D) Percentages of passage 3 and passage 5 UC-MSCs in GO/G1, S, and G2/M cell cycle phases; the data indicate mean \pm SEM of five independent experiments. (E) Representative images of passage 3 (left) and passage 5 (right) UC-MSCs for adipogenesis (top), osteogenesis (middle), and chondrogenesis (bottom); bar $=50 \mu \mathrm{m}$. (F) Flow cytometry analyses of the surface markers of passage 3 (left) and passage 5 (right) UC-MSCs. (G) Percentages of positive cells for CD73, CD90, CD105, CD34, CD45, and HLA-DR between passage 3 and passage 5 UC-MSCs; the data indicate mean \pm SEM of five independent experiments. ns, not significant; SEM, standard error of the mean; UC-MSCs, umbilical cord mesenchymal stem cells. Color images are available online. 
whirlpool-like growth pattern (Fig. 1A). Moreover, both P3 and P5 UC-MSCs showed great and similar growth potential according to results of cell growth curve analysis (Fig. 1B). In the cell cycle analysis, no significant differences were observed in the percentages of different cell cycle stages between P3 and P5 UC-MSCs, as shown in Fig. 1C and $\mathrm{D}$, around $90 \% \mathrm{UC}-\mathrm{MSC}$ were in $\mathrm{G} 0 / \mathrm{G} 1$ phase, $8 \%$ in $\mathrm{S}$ phase, and $2 \%$ in G2/M phase. Culture-expanded UC-MSCs were well characterized based on their ability for differentiation (Fig. 1E) and immunophenotypes (Fig. 1F, G).

After adipogenic differentiation and oil red $\mathrm{O}$ staining, there were obvious red lipid droplets in the induction group compared with the control group. For the osteogenic differentiation, visible nodular cell mass and red calcium deposition were identified in the induction group after alizarin red $\mathrm{S}$ staining. As for chondrogenic differentiation, cell pellets in the induction group were cartilage like and light blue stained, which indicated collagen deposition. Flow cytometry analysis demonstrated that UC-MSCs exhibited over $95 \%$ positive expression of CD73, CD90, and CD105, while $<5 \%$ positive expression of CD34, CD45, and HLA-DR (Fig. 1F). There were no significant differences identified of above cell surface markers between P3 and P5 UC-MSCs (Fig. 1G).

\section{UC-MSCs reduce aneurysmal expansion in the elastase perfusion $A A A$ model}

Animals were injected through tail vein with $1 \times 10^{6}$ UC-MSCs or saline on the first day after aortic perfusion (Fig. 2A). Ultrasonic measurements were performed on days 0,7 , and 14 for all three groups of animals (Fig. 3A). Results showed maximal infrarenal abdominal aortic inner diameters of $1.391 \pm 0.133 \mathrm{~mm}, 1.372 \pm 0.173 \mathrm{~mm}$, and $1.473 \pm$ $0.109 \mathrm{~mm}$ preoperation on day 0 in control, elastase, and elastase+MSC groups, respectively (Fig. 3B). On day 7, a significant increase of maximal inner diameters was observed in elastase group compared to control group (2.257 \pm $0.183 \mathrm{~mm}$ vs. $1.401 \pm 0.160 \mathrm{~mm}$, respectively, $P<0.05$ ), while UC-MSCs $(2.087 \pm 0.111 \mathrm{~mm})$ reduced the maximal inner diameters compared to elastase group $(P<0.05)$ (Fig. 3B). On day 14, UC-MSCs significantly reduced maximal inner diameters compared to elastase group $(2.292 \pm 0.123 \mathrm{~mm}$ vs. $2.635 \pm 0.192 \mathrm{~mm}$, respectively, $P<0.05$ ) (Fig. 3B).

Similar results were also seen in aortic diameter change on day 7 (elastase+MSCs: $0.614 \pm 0.137 \mathrm{~mm}$ vs. elastase: $0.885 \pm 0.280 \mathrm{~mm}, P<0.05$ ) and on day 14 (elastase+MSCs: $0.819 \pm 0.143 \mathrm{~mm}$ vs. elastase: $1.263 \pm 0.267 \mathrm{~mm}, P<0.05$ ) (Fig. 3C). Furthermore, results of percent increase of aortic diameter were consistent with above observations (elastase+ MSCs: $56.228 \% \pm 12.443 \%$ vs. elastase: $94.840 \% \pm 28.474 \%$, $P<0.05$ ) (Fig. 3D). The above ultrasonic results demonstrate that UC-MSCs attenuate aneurysmal expansion in the elastase perfusion AAA model.

\section{UC-MSCs reduce elastin degradation and fragmentation in the elastase perfusion $A A A$ model}

Given the significant aortic diameter differences between elastase+MSC group and elastase group, we further examined

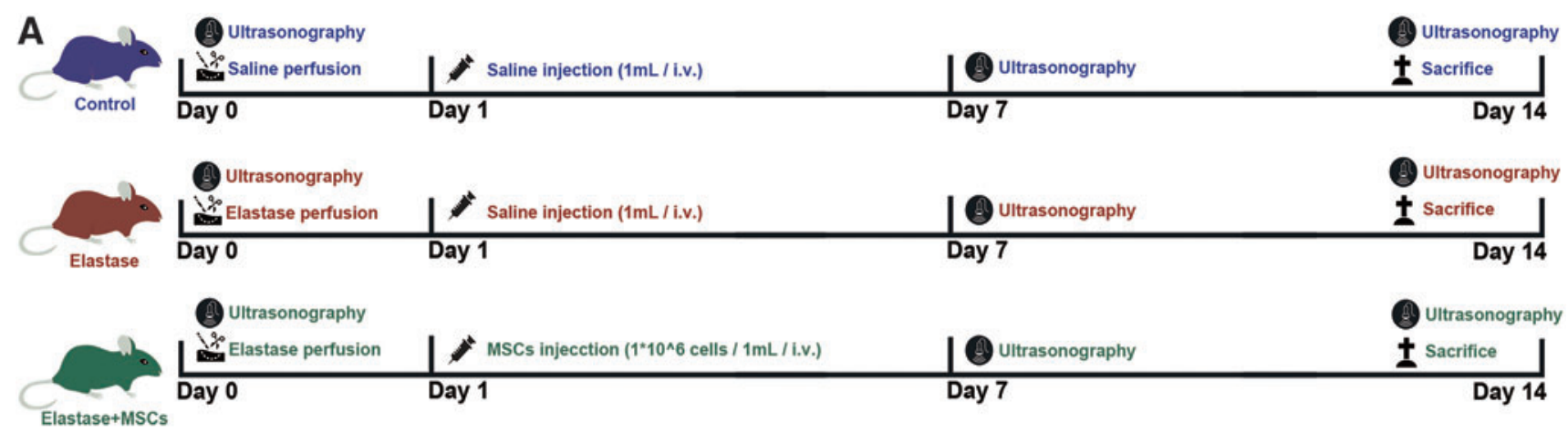

B

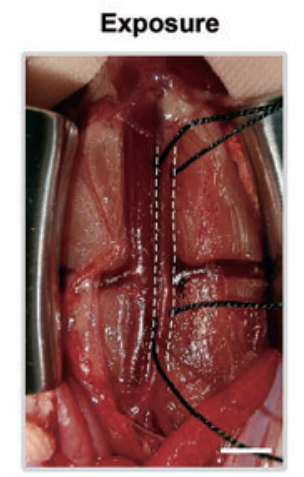

Perfusion

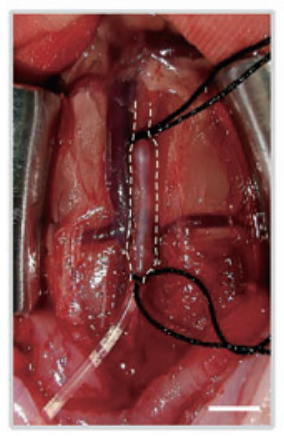

Aneurysm (in situ)

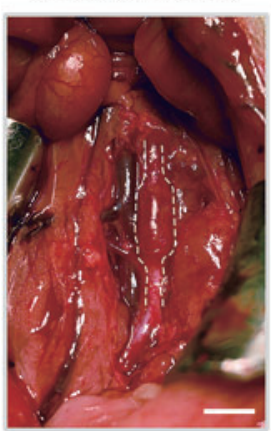

Aneurysm (ex vivo)

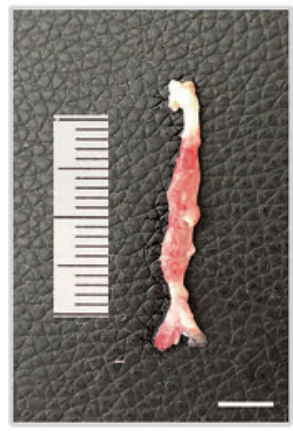

FIG. 2. Study design and establishment of elastase-induced AAA model in SD rats. (A) Flow diagram of study design; animals were randomly divided into three groups: control group, elastase group, and elastase+MSC group; ultrasonography was performed on days 0, 7, and 14; animals were harvested on day 14. (B) Representative images of exposure, elastase perfusion, aneurysmal formation (in situ), and aneurysm formation (in vitro); white dotted lines represent the outline of aorta; bar $=5 \mathrm{~mm}$. AAA, abdominal aortic aneurysm; MSC, mesenchymal stem cell; SD, Sprague-Dawley. Color images are available online. 
A
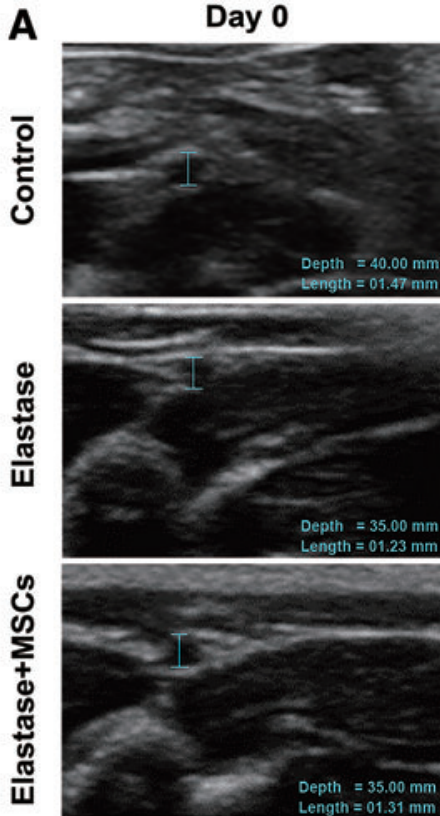

Day 7
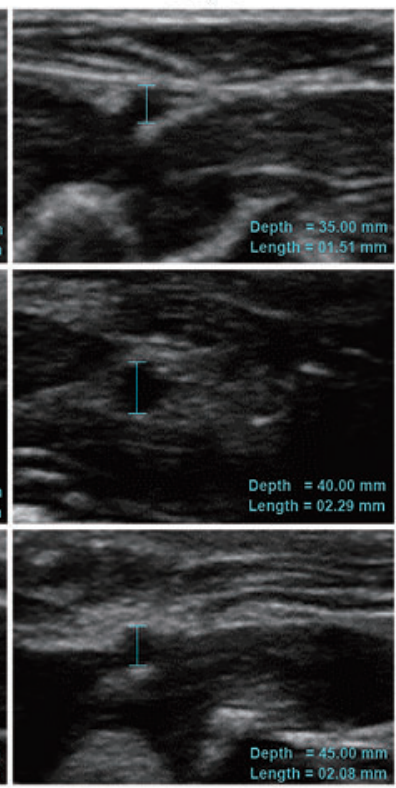

Day 14
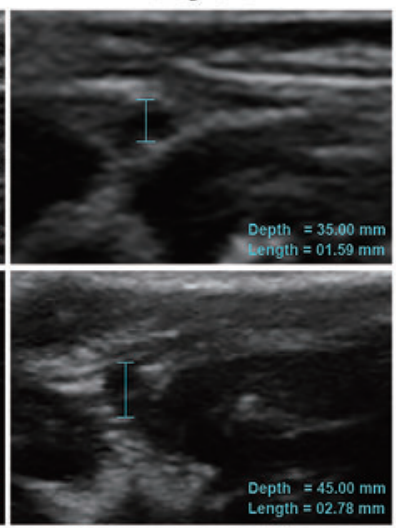
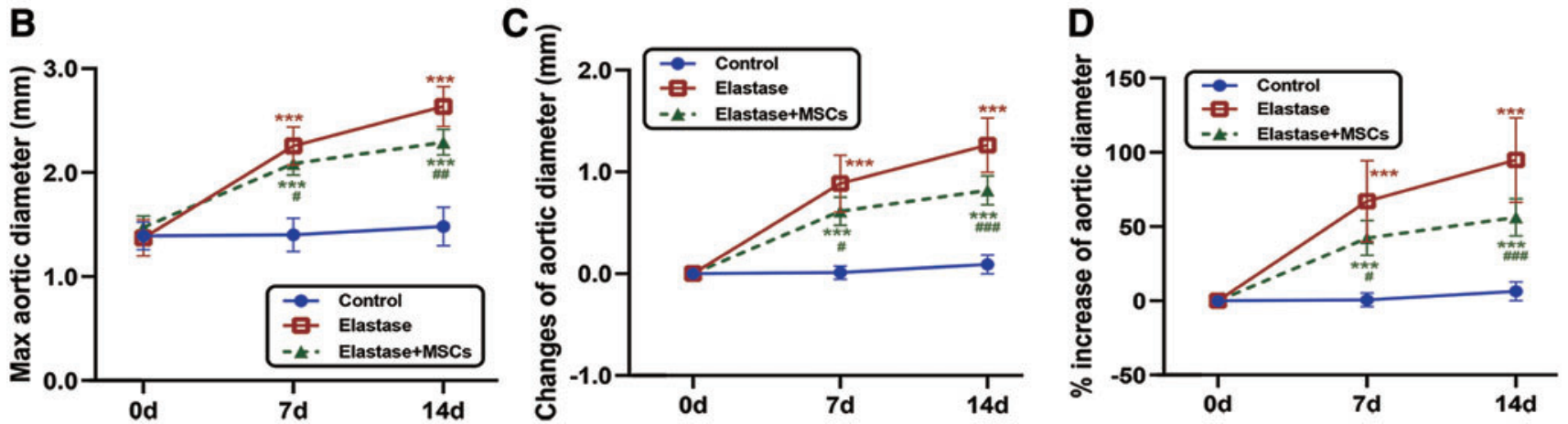

FIG. 3. UC-MSCs reduce aneurysmal expansion in the elastase perfusion AAA model. (A) Representative images for ultrasonography measurements of aortic diameters from three groups of animals on day 0 (left), 7 (middle), and 14 (right). Maximum aortic diameter $(\mathbf{B})$, changes of aortic diameter $(\mathbf{C})$ and percent increase of aortic diameter (D) are shown for the three groups of animals on days 0,7 , and $14 ; n=10$ per group; *** $P<0.001$ compared to control group; ${ }^{\#} P<0.05$, ${ }^{\# \#} P<0.01$, and ${ }^{\# \# \#} P<0.001$ compared to elastase group. Color images are available online.

elastin degradation and fragmentation among three groups of animals on day 14 . Gomori aldehyde fuchsin staining revealed significantly increased level of elastin degradation and fragmentation in elastase group compared to control group $(P<0.05)$, while UC-MSCs significantly reduced elastin degradation and fragmentation compared to elastase group $(P<0.05)$ (Fig. 4). These data demonstrate that UC-MSCs reduce elastin degradation and fragmentation during aneurysm formation.

\section{UC-MSCs promote VSMC contractile phenotypic transition in the elastase perfusion AAA model}

To further elucidate the underlying role of VSMC phenotypic transition between UC-MSC treatment and the attenuation of aneurysm expansion, we examined the expression level of VSMC contractile markers SMA- $\alpha$ and SM-22 and synthetic marker OPN in the medial wall of abdominal aortas on day 14. Results from western blot showed that UC-MSCs effectively restored the expression level of SMA- $\alpha(P<0.05)$ and SM-22 $(P<0.05)$, and attenuated the expression level of OPN $(P<0.05)$ compared with elastase group (Fig. 5A-D). Moreover, UC-MSCs significantly increased the percentage of medial contractile versus synthetic VSMCs compared with elastase group $(P<0.05)$ (Fig. 5E). Similar results were further obtained through IHC (Fig. 6) and immunofluorescence (Fig. 7) staining. These data illustrate that UC-MSCs restore VSMC contractile phenotypic markers within the aortic wall during the aneurysm progression.

\section{UC-MSCs markedly diminish MMPs and TNF- $\alpha$ production in the elastase perfusion AAA model}

VSMCs have been known to be a major source of MMP-2 and MMP-9 production within AAA progression. We found that UC-MSCs contributed to the preservation of VSMC contractile phenotype; thus, we next evaluated the protein level of MMP-2 and MMP-9 with western blot analysis. 

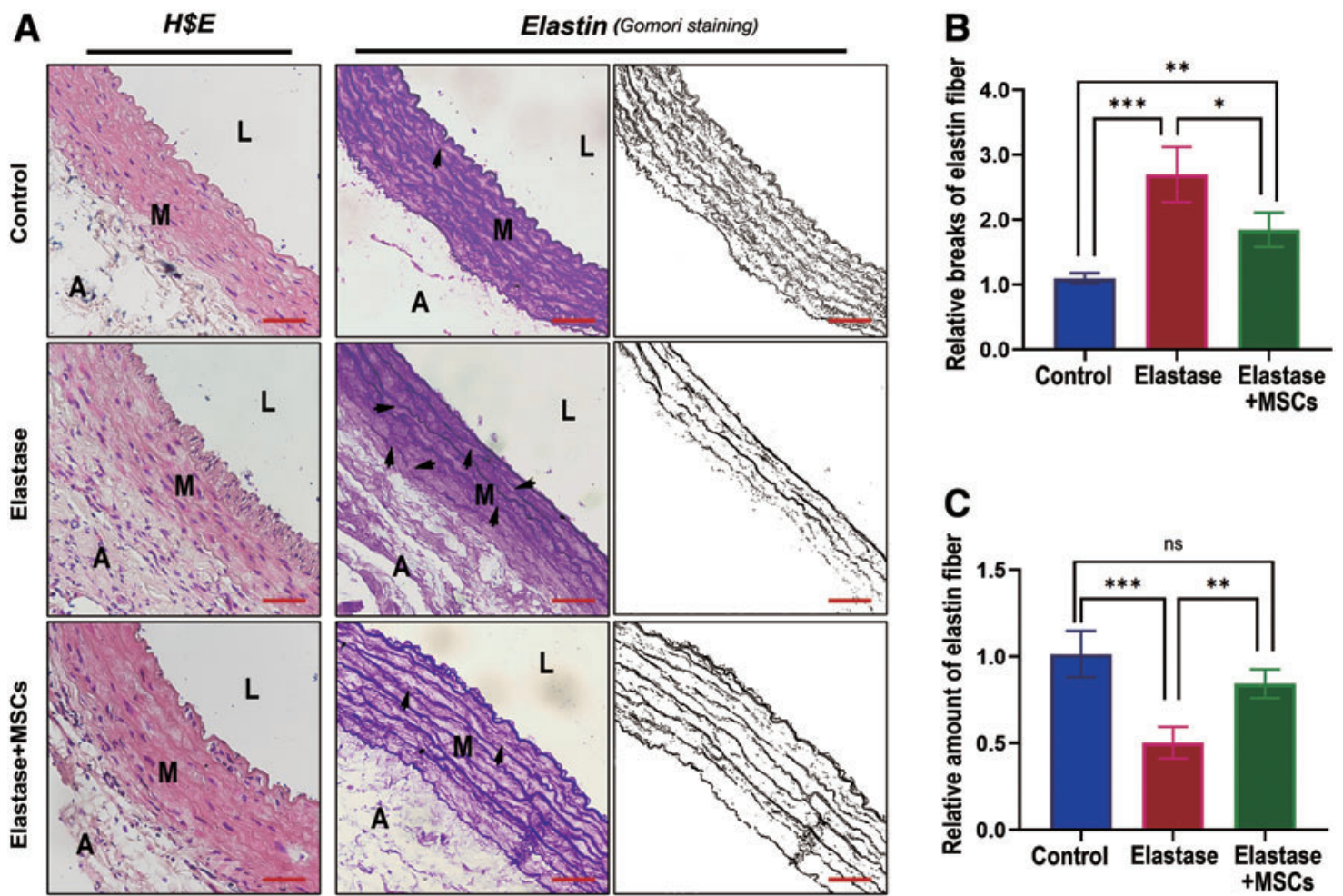

FIG. 4. UC-MSCs reduce elastin degradation and fragmentation in the elastase perfusion AAA model. (A) Representative images of H\&E staining (left) and elastin staining (right) of aortic walls from three groups of animals on day 14; black arrows point to the fragmentation sites of elastin in the aortic wall; bar $=50 \mu \mathrm{m}$. Relative total amount of elastic fibers (B) and breaks of elastic fibers $(\mathbf{C})$ are shown for the three groups of animals; the number of elastin breaks was counted per cross-section; data were normalized to control group; $n=5, * P<0.05$, $* * P<0.01$, and $* * * P<0.001$. A, adventitia of aorta; $\mathrm{H} \& \mathrm{E}$, hematoxylin-eosin; L, lumen of aorta; M, intima media of aorta. Color images are available online.

A
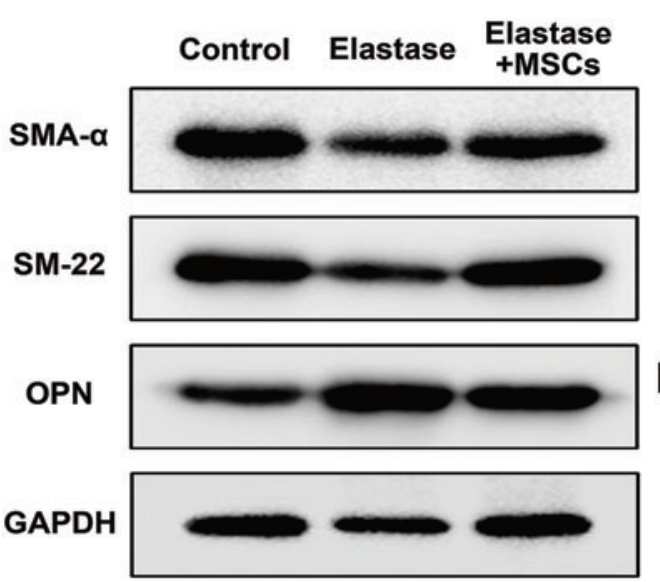

B

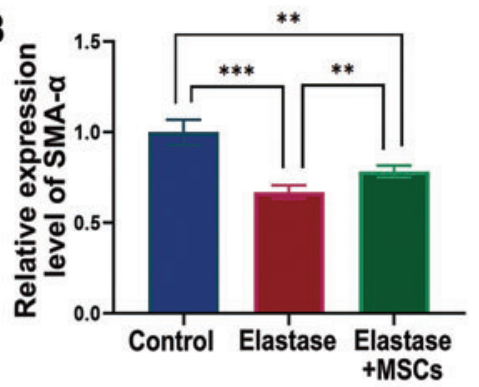

D

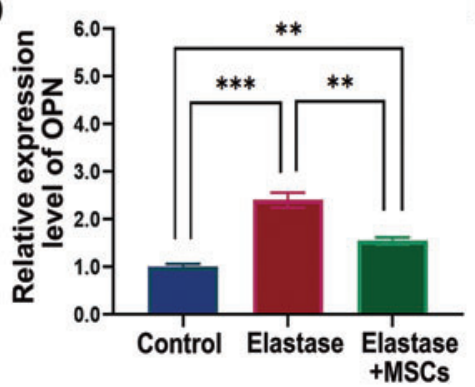

C

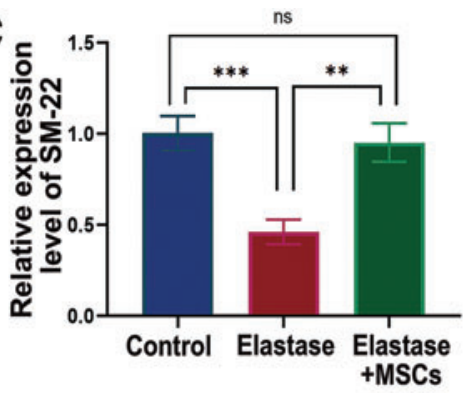

E

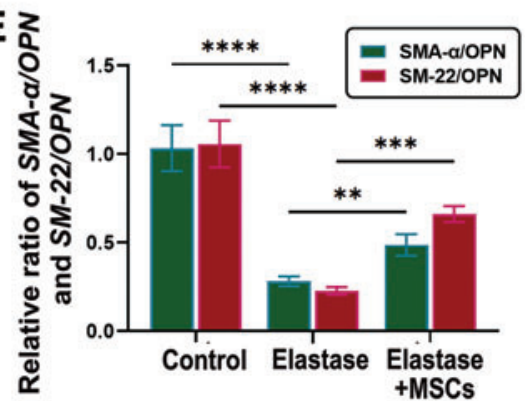

FIG. 5. UC-MSCs promote VSMC contractile phenotypic transition in the elastase perfusion AAA model. (A) Representative western blotting images for VSMC contractile proteins SMA- $\alpha$ and SM-22 and synthetic protein OPN in infrarenal aortas from three groups of animals; each lane represents one group. Bands intensities of SMA- $\alpha$ (B), SM-22 (C), and OPN (D) were normalized to corresponding GAPDH band and expressed as a fraction of control group. (E) The percentage of SMA- $\alpha / \mathrm{OPN}$ and SM-22/OPN are shown. $n=5$. $* * P<0.01 ; * * P<0.001 ; * * * P<0.0001$. VSMC, vascular smooth muscle cell. Color images are available online. 
Indeed, the protein level of MMP-2 and MMP-9 was significantly decreased in UC-MSC-treated group compared with elastase group (Fig. 8A-E). Inflammatory cytokines have been found to be highly correlated with AAA $[1,23,25,26]$. Since UC-MSCs have been known to play a vital role in the regulation of inflammatory reactions during aneurysm formation and progression, we further determined the protein level of TNF- $\alpha$; results demonstrated a significantly lower protein level of TNF- $\alpha$ in UC-MSC-treated group compared with elastase group (Fig. 8A, F). Thus, it appears that UC-MSCs contribute to the suppression of MMPs and deterioration of inflammatory reactions within the aneurysm wall.

\section{Discussion}

In this study, we used porcine pancreatic elastase intraluminal perfusion model to investigate the therapeutic efficacy of intravenous UC-MSC infusion in AAA. Intraluminal perfusion with elastase model, one of the most widely used experimental models of AAA in vivo, was first introduced by Anidjar et al. in Wistar rats [27], which simulates the patho- logical characteristics of medial degeneration and leukocyte infiltration in the occurrence and development of human AAA $[28,29]$. In this study, we established the elastase-induced AAA model in SD rats, and the abdominal aortas were morphologically aneurysmal on the 14th postoperative day, which is consist with previous investigations [24,30].

According to the minimal criteria stated by the International Society for Cellular Therapy (ISCT), MSCs are characterized by plastic-adherent, high expression of CD73, CD90, and CD105, and low expression of CD34, CD45, and HLA-DR, abilities of adipogenesis, osteogenesis, and chondrogenesis [31]. In this study, the explant method [32] was implemented to obtain UC-MSCs from Wharton jelly in vitro and cells in passages 3 and 5 were utilized in the following experiments. Results of UC-MSC characterization (Fig. 1) showed that cells used in this study were in line with the ISCT standards and were eligible to follow-up experiments.

Phenotypic transition of VSMCs during AAA initiation and progression has been demonstrated by previous literatures $[9,33]$. To be consistent with previous studies, we demonstrated in this study that the expression level of
A

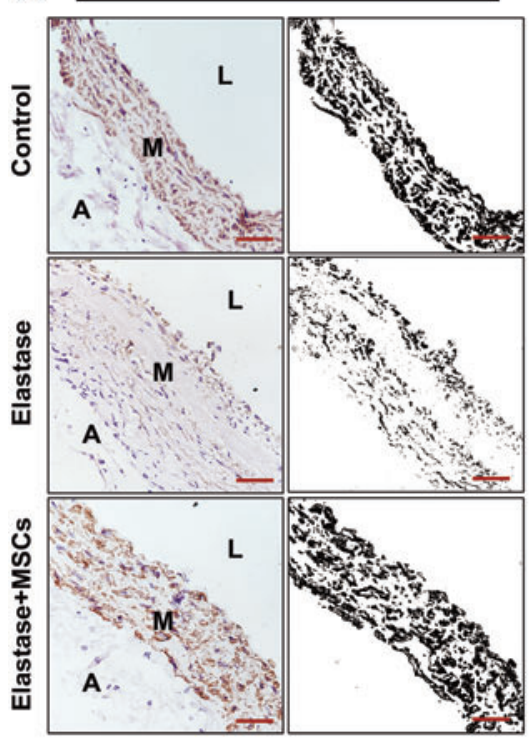

B

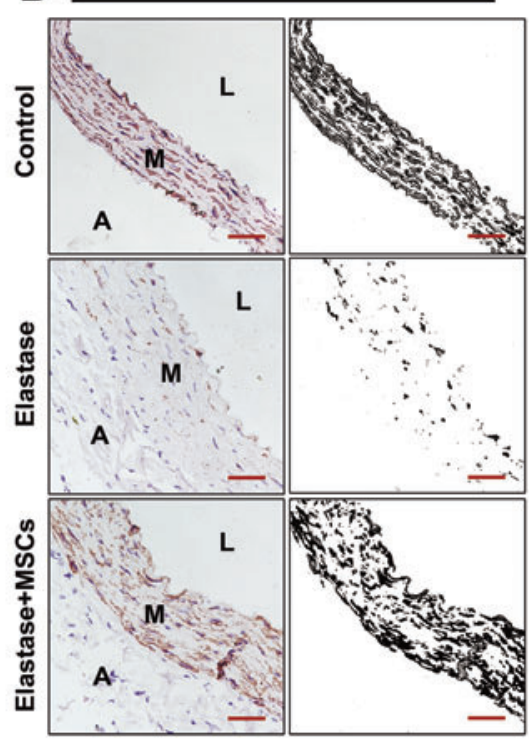

C

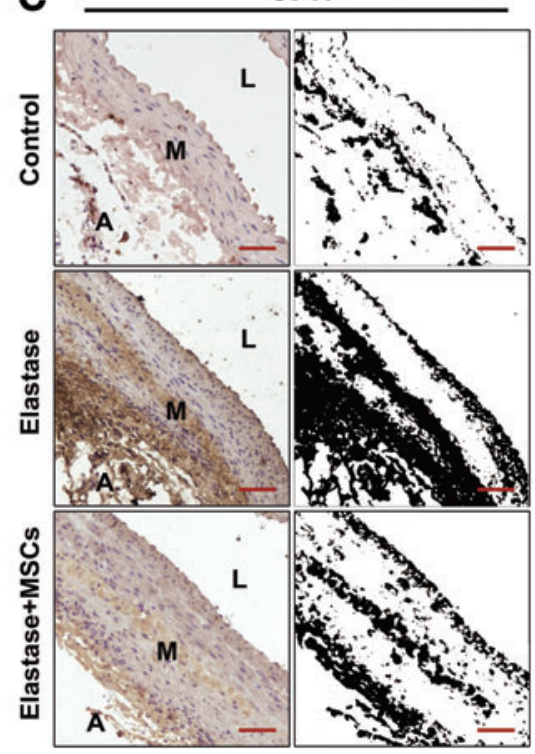

D

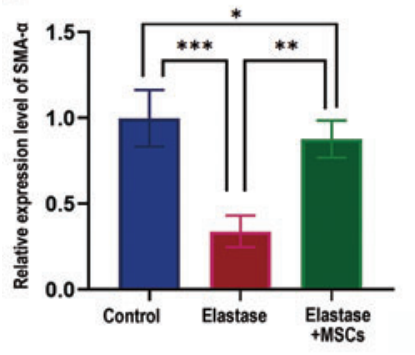

E

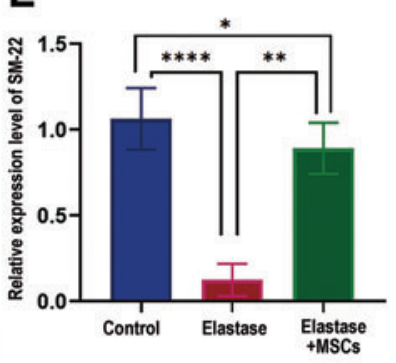

$\mathbf{F}$

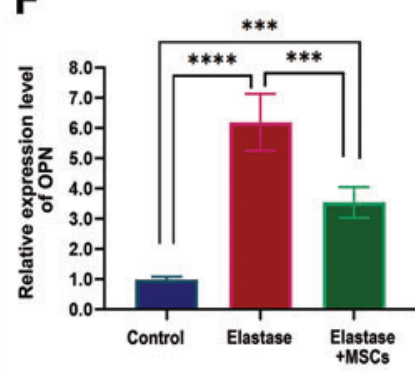

G

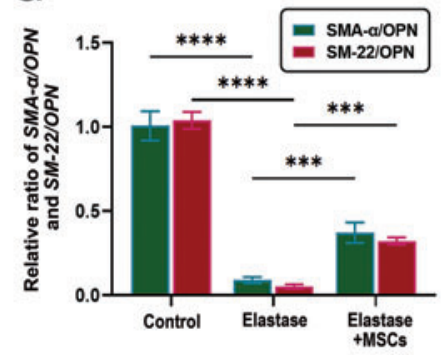

FIG. 6. UC-MSCs promote VSMC contractile phenotypic transition in the elastase perfusion AAA model. Representative images of immunohistochemistry staining for SMA- $\alpha$ (left) (A), SM-22 (middle) (B), and OPN (right) (C) in the medial aortic walls from three groups of animals on day 14 ; bar $=50 \mu \mathrm{m}$. Measurements of SMA- $\alpha$ (D), SM-22 (E), and OPN (F) per cross-section are shown for the three groups of animals. (G) The percentage of SMA- $\alpha / O P N$ and SM-22/OPN in the medial wall is shown; data are normalized to control group; $n=5, * P<0.05$, $* * P<0.01$, $* * * P<0.001$, and $* * * * P<0.0001$. Color images are available online. 

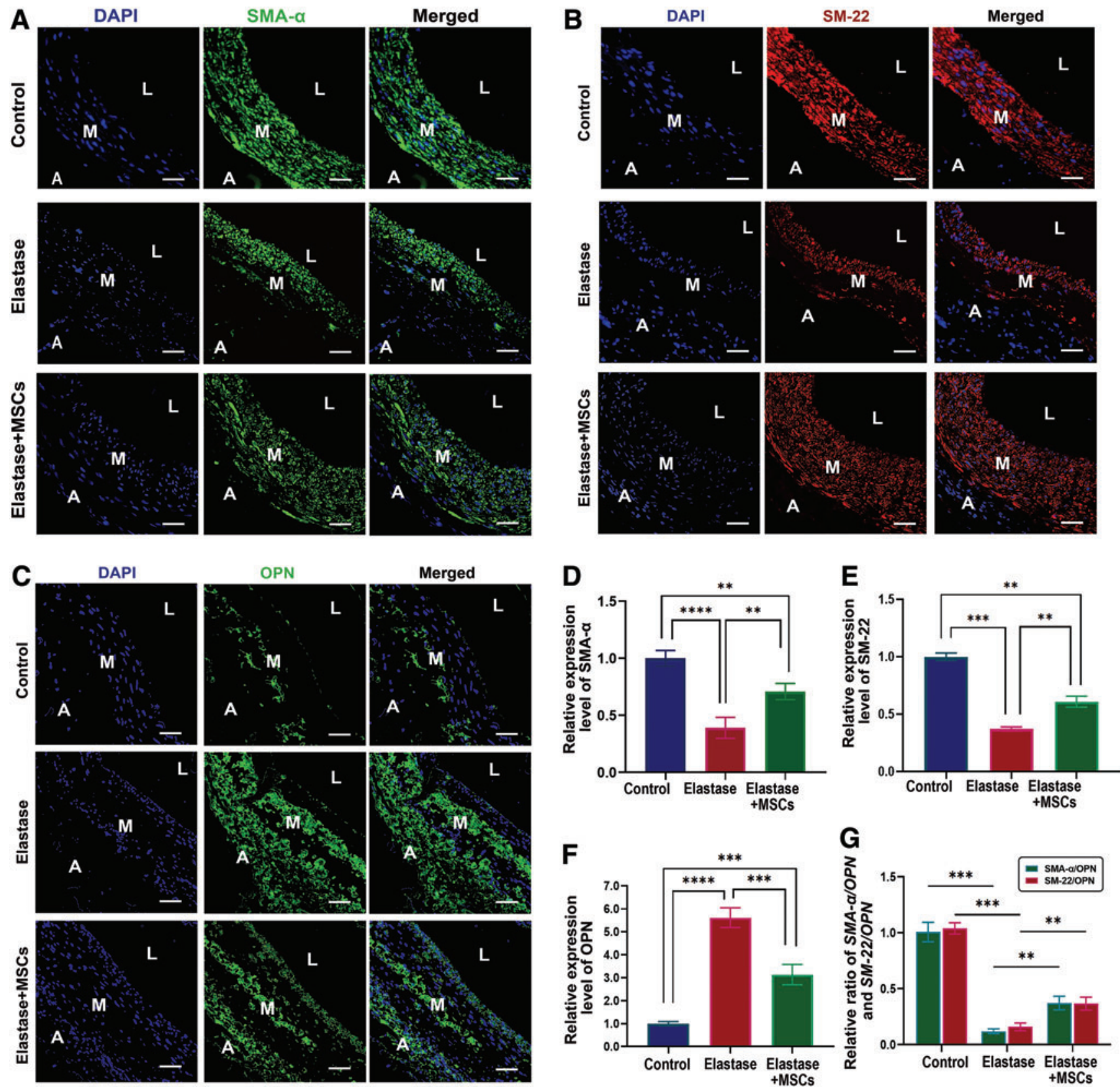

FIG. 7. UC-MSCs promote VSMC contractile phenotypic transition in the elastase perfusion AAA model. Representative images of immunofluorescence staining for DAPI (nuclei marker, blue) and SMA- $\alpha$ (green) (A), SM-22 (red) (B), and OPN (green) (C) with merged images in the aortic walls from three groups of animals on day 14; bar $=50 \mu \mathrm{m}$. Measurements of SMA- $\alpha$ (D), SM-22 (E), and OPN (F) per cross-section are shown for the three groups of animals. (G) The percentage of SMA- $\alpha /$ OPN and SM-22/OPN in the medial wall is shown; data were normalized to control group; $n=5, * * P<0.01$, $* * * P<0.001$, and $* * * * P<0.0001$. Color images are available online.

contractile proteins SMA- $\alpha$ and SM-22 was reduced in the process of AAA progression, while the expression level of synthetic protein OPN was increased. To further investigate the effect of UC-MSCs on VSMC phenotypic transition in AAA, we intravenously injected $1 \times 10^{6}$ UC-MSCs into AAA models and detected the protein level of SMA- $\alpha$, SM22, and OPN. Results showed that UC-MSCs preserved and restored the contractile phenotype of VSMCs, which further contributes to the attenuation of AAA progression. Compared to previous studies investigating the beneficial effect of MSCs in AAA [14-20,34], our research revealed that
UC-MSCs could perform therapeutic effect in AAA through modulating VSMC phenotype.

UC-MSCs were reported to be efficient in various diseases through their paracrine effects [35,36]. One cytokine profiling study found that UC-MSCs showed significantly higher secretion level of TGF- $\beta$, a strong promotor of VSMC contractile phenotypic transition [37], compared to adipose tissue- and amniotic membrane-derived MSCs [38]. Thus, we conservatively hypothesize that UC-MSCs might, in part, promote VSMC contractile phenotype through directly secreting TGF- $\beta$ in AAA, although needs to be further elucidated. 


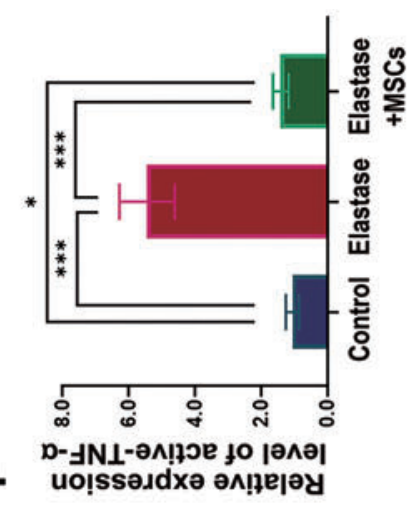

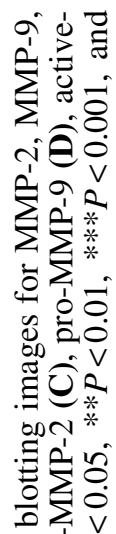
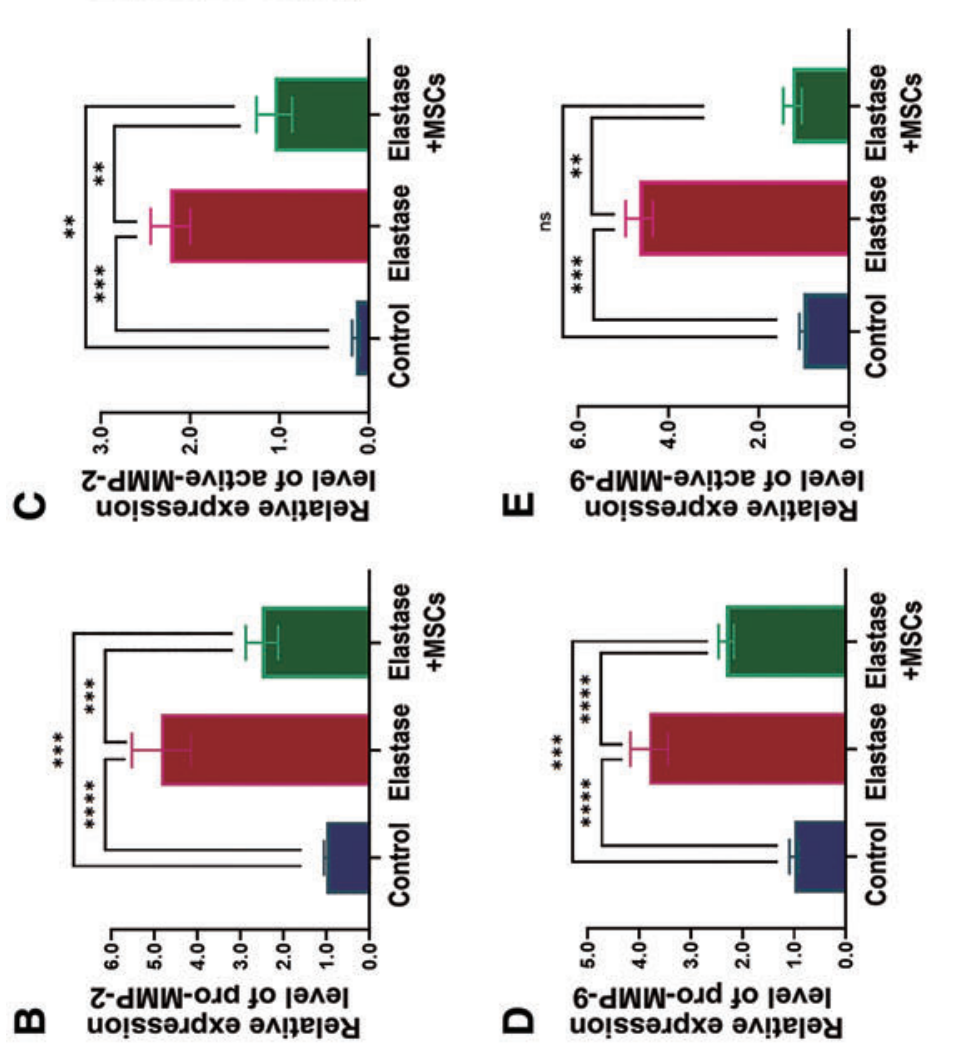

है

号 ชิ จย 줄 氖这

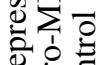
늘 응 ङ 응

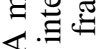
$\sum_{0} \pi$ 《 ธี ซ 它完 ¿ क

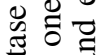
芯芯元 휴음 을 른핀 들 원 氜 을 이 o. 유 증 㞱寻 눙 $\exists \circ \circ$ 结吾 $\sum$ to. N $\sum 0$ 궁 도름쥬 施 氙氙 츔웜 过氙

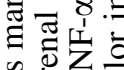
的垙

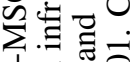
ㄴ. 엉잉 $\infty$ Zो

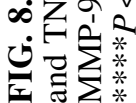


The normal biomechanical properties of arterial walls are partially maintained by the complex spatial structure consisting of elastic fibers and their interactions with collagen and VSMCs [39]. Degradation and fragmentation of elastic fibers caused by MMPs are thought to contribute to the initiation and progression of AAA [39-41]. Several promising studies have found MSCs derived from bone marrow [14,15,34], adipose tissue [17,18], placenta [16], or umbilical cord tissue [19] can inhibit the expansion of aneurysmal sac, and reduce the protein level of MMP-2 and MMP-9 and elastin degradation within the arterial wall. In this study, we measured the maximum aortic diameter at 0,7 , and 14 days with ultrasound technique, assessed the degree of elastin degradation and fragmentation at 14 days by elastin staining, and evaluated the protein level of MMP-2 and MMP-9 through western blot.

Despite differences in cell origin, route of administration, number of cells, and animal models, our results were similar with previous investigations that UC-MSCs could attenuate aneurysm expansion, reduce elastin degradation and fragmentation, and inhibit MMP-2 and MMP-9 activities. MMP2 is reported to be predominantly derived from VSMCs during AAA initiation and progression [42-44]; alteration of MMP-2 secretion level is part of the process of VSMC phenotypic regulation [45]. Interestingly, in this study, we revealed the protective effect of UC-MSCs on VSMC contractile phenotype, which may be closely associated with the decreased expression level of MMP-2.

Inflammatory cells and cytokines have been determined to be involved in the pathogenesis of AAA $[25,46]$. TNF- $\alpha$ is primarily produced by monocyte macrophage system in AAA [26,47]. Previous studies have revealed that TNF- $\alpha$ could induce VSMCs from contractile to synthetic phenotype through the downregulation of VSMC-specific contractile genes $[48,49]$. In this study, we demonstrated that UC-MSCs suppress TNF- $\alpha$ expression during AAA initiation and progression, which implies that UC-MSCs might promote the phenotypic transition of VSMCs through indirect pathways, such as downregulating the expression level of TNF- $\alpha$. In addition, VSMCs are found to be a source of TNF- $\alpha$ secretion in inflammatory conditions [50,51]. Thus, UC-MSC-induced contractile VSMCs might further suppress TNF- $\alpha$ secretion, which, taken together, forms a positive feedback circuit.

In addition to stem cells from various origins, non-stem cell-based cellular therapy for AAA such as VSMCs is another important candidate cell source. Allaire et al. [52] have determined that the VSMC endovascular seeding restores the healing capabilities of proteolytically injured ECM in aneurysmal aortas, and stops aneurysmal expansion. In another study comparing the therapeutic efficacy of bone marrow-derived mesenchymal stem cells (BM-MSCs) and VSMCs in an already-formed aortic aneurysm xenograft model, Allaire and colleagues [53] found that BM-MSCs stabilize already-formed aortic aneurysms more efficiently than VSMCs. Moreover, Ramamurthi and colleagues [5457] have demonstrated the proelastogenic properties of bone marrow MSC-derived smooth muscle cells and highlighted the future application of in-vitro induced smooth muscle cells in AAA management. Although results of MSCs from multiple tissue sources and in vitro cultured or induced VSMCs in the treatment of AAA are promising in preclinical studies, further investigations are still needed.
In this study, a single dose of $1 \times 10^{6}$ UC-MSCs were injected intravenously on the 1 st postoperative day, which gave rise to considerations about optimal injecting cell amount, along with the frequency and timing of injection. Compared to studies with local implantation of MSCs $[15,20]$, intravenous injection of MSCs represents the minimum invasive systematic route for cellular therapy, and has been previously shown to be effective in different AAA models [16,18,34]. In addition, a randomized controlled trail investigating MSCs in small human AAA also applied intravenous administration [58], which further arise the clinical convenience and feasibility of intravenous administration.

Intravenous injected BM-MSCs were observed in the infrarenal aorta segments at 2 weeks after administration, as reported by Yamawaki-Ogata et al. [34], which addressed the in vivo mobilization and local differentiation of MSCs in AAA. However, in this study, we did not evaluate the distribution of UC-MSCs in the infrarenal aorta tissue. Moreover, this study established the biological effect of UCMSCs promoting or preserving VSMC contractile phenotype in AAA, but the underlying mechanisms have not been fully elucidated and need to be further investigated.

\section{Conclusion}

In summary, this study demonstrates that intravenous injection of UC-MSCs attenuates elastase-induced AAA initiation and progression through restoration and/or preservation of VSMC contractile phenotype, impaired activity of MMP-2 and MMP-9, reduced level of elastin degradation and fragmentation, and downregulated expression of inflammatory cytokine TNF- $\alpha$. Taken together, the findings presented herein provide a theoretical and experimental basis for the future application of UC-MSCs and other stem cell-based therapies for AAA.

\section{Author Disclosure Statement}

The authors declare no competing financial or other conflict of interests.

\section{Funding Information}

This study was supported by the National Natural Science Foundation of China (nos. 81770488 and 81974049).

\section{References}

1. Golledge J. (2019). Abdominal aortic aneurysm: update on pathogenesis and medical treatments. Nat Rev Cardiol 16: 225-242.

2. Powell JT, MJ Sweeting, P Ulug, JD Blankensteijn, FA Lederle, JP Becquemin and RM Greenhalgh; EVAR-1, DREAM, OVER and ACE Trialists. (2017). Meta-analysis of individual-patient data from EVAR-1, DREAM, OVER and ACE trials comparing outcomes of endovascular or open repair for abdominal aortic aneurysm over 5 years. $\mathrm{Br}$ J Surg 104:166-178.

3. Lederle FA, TC Kyriakides, KT Stroupe, JA Freischlag, FT Padberg, Jr., JS Matsumura, Z Huo and GR Johnson; OVER Veterans Affairs Cooperative Study Group. (2019). Open versus endovascular repair of abdominal aortic aneurysm. N Engl J Med 380:2126-2135. 
4. Patel R, MJ Sweeting, JT Powell and RM Greenhalgh; EVAR Trial Investigators. (2016). Endovascular versus open repair of abdominal aortic aneurysm in 15-years' follow-up of the UK endovascular aneurysm repair trial 1 (EVAR trial 1): a randomised controlled trial. Lancet 388:2366-2374.

5. van Schaik TG, KK Yeung, HJ Verhagen, JL de Bruin, M van Sambeek, R Balm, CJ Zeebregts, JA van Herwaarden and JD Blankensteijn; DREAM Trial Participants. (2017). Long-term survival and secondary procedures after open or endovascular repair of abdominal aortic aneurysms. J Vasc Surg 66:1379-1389.

6. Lindeman JH and JS Matsumura. (2019). Pharmacologic management of aneurysms. Circ Res 124:631-646.

7. Sakalihasan N, JB Michel, A Katsargyris, H Kuivaniemi, JO Defraigne, A Nchimi, JT Powell, K Yoshimura and R Hultgren. (2018). Abdominal aortic aneurysms. Nat Rev Dis Primers 4:34.

8. Frismantiene A, M Philippova, P Erne and TJ Resink. (2018). Smooth muscle cell-driven vascular diseases and molecular mechanisms of VSMC plasticity. Cell Signal 52:48-64.

9. Ailawadi G, CW Moehle, H Pei, SP Walton, Z Yang, IL Kron, CL Lau and GK Owens. (2009). Smooth muscle phenotypic modulation is an early event in aortic aneurysms. J Thorac Cardiovasc Surg 138:1392-1399.

10. Hulsmans $M$ and M Nahrendorf. (2020). Proliferative, degradative smooth muscle cells promote aortic disease. J Clin Invest 130:1096-1098.

11. Petsophonsakul P, M Furmanik, R Forsythe, M Dweck, GW Schurink, E Natour, C Reutelingsperger, M Jacobs, B Mees and L Schurgers. (2019). Role of vascular smooth muscle cell phenotypic switching and calcification in aortic aneurysm formation. Arterioscler Thromb Vasc Biol 39: 1351-1368.

12. Allahverdian S, C Chaabane, K Boukais, GA Francis and ML Bochaton-Piallat. (2018). Smooth muscle cell fate and plasticity in atherosclerosis. Cardiovasc Res 114:540-550.

13. Bennett MR, S Sinha and GK Owens. (2016). Vascular smooth muscle cells in atherosclerosis. Circ Res 118:692-702.

14. Fu XM, A Yamawaki-Ogata, H Oshima, Y Ueda, A Usui and Y Narita. (2013). Intravenous administration of mesenchymal stem cells prevents angiotensin II-induced aortic aneurysm formation in apolipoprotein E-deficient mouse. $\mathbf{J}$ Transl Med 11:175.

15. Hashizume R, A Yamawaki-Ogata, Y Ueda, WR Wagner and Y Narita. (2011). Mesenchymal stem cells attenuate angiotensin II-induced aortic aneurysm growth in apolipoprotein E-deficient mice. J Vasc Surg 54:1743-1752.

16. Sharma AK, G Lu, A Jester, WF Johnston, Y Zhao, VA Hajzus, MR Saadatzadeh, G Su, CM Bhamidipati, et al. (2012). Experimental abdominal aortic aneurysm formation is mediated by IL-17 and attenuated by mesenchymal stem cell treatment. Circulation 126:S38-S45.

17. Blose KJ, TL Ennis, B Arif, JS Weinbaum, JA Curci and DA Vorp. (2014). Periadventitial adipose-derived stem cell treatment halts elastase-induced abdominal aortic aneurysm progression. Regen Med 9:733-741.

18. Xie J, TJ Jones, D Feng, TG Cook, AA Jester, R Yi, YT Jawed, C Babbey, KL March and MP Murphy. (2017). Human adipose-derived stem cells suppress elastase-induced murine abdominal aortic inflammation and aneurysm expansion through paracrine factors. Cell Transplant 26:173-189.

19. Sharma AK, MD Salmon, G Lu, G Su, NH Pope, JR Smith, ML Weiss and GR Upchurch, Jr. (2016). Mesenchymal stem cells attenuate NADPH oxidase-dependent high mobility group box 1 production and inhibit abdominal aortic aneurysms. Arterioscler Thromb Vasc Biol 36:908-918.

20. Parvizi M, AH Petersen, C van Spreuwel-Goossens, S Kluijtmans and MC Harmsen. (2018). Perivascular scaffolds loaded with adipose tissue-derived stromal cells attenuate development and progression of abdominal aortic aneurysm in rats. J Biomed Mater Res A 106:2494-2506.

21. Parvizi M and MC Harmsen. (2015). Therapeutic prospect of adipose-derived stromal cells for the treatment of abdominal aortic aneurysm. Stem Cells Dev 24:1493-1505.

22. Deng Y, S Yi, G Wang, J Cheng, Y Zhang, W Chen, Y Tai, S Chen, G Chen, et al. (2014). Umbilical cord-derived mesenchymal stem cells instruct dendritic cells to acquire tolerogenic phenotypes through the IL-6-mediated upregulation of SOCS1. Stem Cells Dev 23:2080-2092.

23. Park M, S Nepali and H Lew. (2020). Isolation and characterization of extraocular muscle-derived muscle progenitor cells from normal and Graves' orbitopathy patients. Stem Cells Dev 29:353-363.

24. Ren J, Y Han, T Ren, H Fang, X Xu, Y Lun, H Jiang, S Xin and J Zhang. (2020). AEBP1 promotes the occurrence and development of abdominal aortic aneurysm by modulating inflammation via the NF-kappaB pathway. J Atheroscler Thromb 27:255-270.

25. Wang SK, LA Green, AR Gutwein, NA Drucker, RL Motaganahalli, AK Gupta, A Fajardo and MP Murphy. (2018). Description of human AAA by cytokine and immune cell aberrations compared to risk-factor matched controls. Surgery 164:354-358.

26. Potteaux S and A Tedgui. (2015). Monocytes, macrophages and other inflammatory mediators of abdominal aortic aneurysm. Curr Pharm Des 21:4007-4015.

27. Anidjar S, JL Salzmann, D Gentric, P Lagneau, JP Camilleri and JB Michel. (1990). Elastase-induced experimental aneurysms in rats. Circulation 82:973-981.

28. Lysgaard Poulsen J, J Stubbe and JS Lindholt. (2016). Animal models used to explore abdominal aortic aneurysms: a systematic review. Eur J Vasc Endovasc Surg 52: 487-499.

29. Senemaud J, G Caligiuri, H Etienne, S Delbosc, JB Michel and R Coscas. (2017). Translational relevance and recent advances of animal models of abdominal aortic aneurysm. Arterioscler Thromb Vasc Biol 37:401-410.

30. Yao F, Z Yao, T Zhong, J Zhang, T Wang, B Zhang, Q He, L Ding and B Yang. (2019). Imatinib prevents elastaseinduced abdominal aortic aneurysm progression by regulating macrophage-derived MMP9. Eur J Pharmacol 860: 172559.

31. Dominici M, K Le Blanc, I Mueller, I Slaper-Cortenbach, F Marini, D Krause, R Deans, A Keating, D Prockop and E Horwitz. (2006). Minimal criteria for defining multipotent mesenchymal stromal cells. The International Society for Cellular Therapy position statement. Cytotherapy 8:315-317.

32. Marmotti A, S Mattia, M Bruzzone, S Buttiglieri, A Risso, DE Bonasia, D Blonna, F Castoldi, R Rossi, et al. (2012). Minced umbilical cord fragments as a source of cells for orthopaedic tissue engineering: an in vitro study. Stem Cells Int 2012:326813.

33. Zhong L, X He, X Si, H Wang, B Li, Y Hu, M Li, X Chen, W Liao, Y Liao and J Bin. (2019). SM22alpha (smooth muscle 22alpha) prevents aortic aneurysm formation by inhibiting smooth muscle cell phenotypic switching through suppressing reactive oxygen species/NF-kappaB (nuclear factor-kappaB). Arterioscler Thromb Vasc Biol 39:e10-e25. 
34. Yamawaki-Ogata A, X Fu, R Hashizume, KL Fujimoto, Y Araki, H Oshima, Y Narita and A Usui. (2014). Therapeutic potential of bone marrow-derived mesenchymal stem cells in formed aortic aneurysms of a mouse model. Eur J Cardiothorac Surg 45:e156-e165.

35. Saulnier N, E Viguier, E Perrier-Groult, C Chenu, E Pillet, T Roger, S Maddens and C Boulocher. (2015). Intra-articular administration of xenogeneic neonatal mesenchymal stromal cells early after meniscal injury down-regulates metalloproteinase gene expression in synovium and prevents cartilage degradation in a rabbit model of osteoarthritis. Osteoarthritis Cartilage 23:122-133.

36. Zhang Y, Z Zhu, K Hua, L Yao, Y Liu and J Ding. (2018). Umbilical cord-derived mesenchymal stem cell transplantation in vaginal replacement in vitro and in a rat model. Am J Transl Res 10:3762-3772.

37. Guo X and SY Chen. (2012). Transforming growth factorbeta and smooth muscle differentiation. World J Biol Chem 3:41-52.

38. Dabrowski FA, A Burdzinska, A Kulesza, A Sladowska, A Zolocinska, K Gala, L Paczek and M Wielgos. (2017). Comparison of the paracrine activity of mesenchymal stem cells derived from human umbilical cord, amniotic membrane and adipose tissue. J Obstet Gynaecol Res 43:1758-1768.

39. Hellenthal FA, WA Buurman, WK Wodzig and GW Schurink. (2009). Biomarkers of AAA progression. Part 1: extracellular matrix degeneration. Nat Rev Cardiol 6:464-474.

40. Campa JS, RM Greenhalgh and JT Powell. (1987). Elastin degradation in abdominal aortic aneurysms. Atherosclerosis 65:13-21.

41. Basalyga DM, DT Simionescu, W Xiong, BT Baxter, BC Starcher and NR Vyavahare. (2004). Elastin degradation and calcification in an abdominal aorta injury model: role of matrix metalloproteinases. Circulation 110:3480-3487.

42. Crowther M, S Goodall, JL Jones, PR Bell and MM Thompson. (2000). Increased matrix metalloproteinase 2 expression in vascular smooth muscle cells cultured from abdominal aortic aneurysms. J Vasc Surg 32:575-583.

43. Lesauskaite V, MC Epistolato, M Castagnini, S Urbonavicius and $P$ Tanganelli. (2006). Expression of matrix metalloproteinases, their tissue inhibitors, and osteopontin in the wall of thoracic and abdominal aortas with dilatative pathology. Hum Pathol 37:1076-1084.

44. Goodall S, M Crowther, DM Hemingway, PR Bell and MM Thompson. (2001). Ubiquitous elevation of matrix metalloproteinase- 2 expression in the vasculature of patients with abdominal aneurysms. Circulation 104:304-309.

45. Risinger GM, Jr., TS Hunt, DL Updike, EC Bullen and EW Howard. (2006). Matrix metalloproteinase-2 expression by vascular smooth muscle cells is mediated by both stimulatory and inhibitory signals in response to growth factors. $\mathrm{J}$ Biol Chem 281:25915-25925.

46. Dale MA, MK Ruhlman and BT Baxter. (2015). Inflammatory cell phenotypes in AAAs: their role and potential as targets for therapy. Arterioscler Thromb Vasc Biol 35:1746-1755.

47. Raffort J, F Lareyre, M Clement, R Hassen-Khodja, G Chinetti and Z Mallat. (2017). Monocytes and macrophages in abdominal aortic aneurysm. Nat Rev Cardiol 14:457-471.

48. Choi S, M Park, J Kim, W Park, S Kim, DK Lee, JY Hwang, J Choe, MH Won, et al. (2018). TNF-alpha elicits phenotypic and functional alterations of vascular smooth muscle cells by miR-155-5p-dependent down-regulation of cGMP-dependent kinase 1. J Biol Chem 293:14812-14822.
49. Ali MS, RM Starke, PM Jabbour, SI Tjoumakaris, LF Gonzalez, RH Rosenwasser, GK Owens, WJ Koch, NH Greig and AS Dumont. (2013). TNF-alpha induces phenotypic modulation in cerebral vascular smooth muscle cells: implications for cerebral aneurysm pathology. J Cereb Blood Flow Metab 33:1564-1573.

50. Liu N, J Liu, Y Ji, P Lu, C Wang and F Guo. (2011). C-reactive protein induces TNF-alpha secretion by $\mathrm{p} 38$ MAPK-TLR4 signal pathway in rat vascular smooth muscle cells. Inflammation 34:283-290.

51. Warner SJ and P Libby. (1989). Human vascular smooth muscle cells. Target for and source of tumor necrosis factor. J Immunol 142:100-109.

52. Allaire E, B Muscatelli-Groux, AM Guinault, C Pages, A Goussard, C Mandet, P Bruneval, D Melliere and JP Becquemin. (2004). Vascular smooth muscle cell endovascular therapy stabilizes already developed aneurysms in a model of aortic injury elicited by inflammation and proteolysis. Ann Surg 239:417-427.

53. Schneider F, F Saucy, R de Blic, J Dai, F Mohand, H Rouard, JB Ricco, JP Becquemin, M Gervais and E Allaire. (2013). Bone marrow mesenchymal stem cells stabilize already-formed aortic aneurysms more efficiently than vascular smooth muscle cells in a rat model. Eur J Vasc Endovasc Surg 45:666-672.

54. Dahal S, T Broekelman, RP Mecham and A Ramamurthi. (2018). Maintaining elastogenicity of mesenchymal stem cell-derived smooth muscle cells in two-dimensional culture. Tissue Eng Part A 24:979-989.

55. Dahal S, G Swaminathan, S Carney, T Broekelmann, R Mecham and A Ramamurthi. (2020). Pro-elastogenic effects of mesenchymal stem cell derived smooth muscle cells in a 3D collagenous milieu. Acta Biomater 105:180-190.

56. Swaminathan G, VS Gadepalli, I Stoilov, RP Mecham, RR Rao and A Ramamurthi. (2017). Pro-elastogenic effects of bone marrow mesenchymal stem cell-derived smooth muscle cells on cultured aneurysmal smooth muscle cells. J Tissue Eng Regen Med 11:679-693.

57. Swaminathan G, I Stoilov, T Broekelmann, R Mecham and A Ramamurthi. (2018). Phenotype-based selection of bone marrow mesenchymal stem cell-derived smooth muscle cells for elastic matrix regenerative repair in abdominal aortic aneurysms. J Tissue Eng Regen Med 12:e60-e70.

58. Wang SK, LA Green, AR Gutwein, NA Drucker, RL Motaganahalli, A Fajardo, CM Babbey and MP Murphy. (2018). Rationale and design of the ARREST trial investigating mesenchymal stem cells in the treatment of small abdominal aortic aneurysm. Ann Vasc Surg 47:230-237.

Address correspondence to: Prof. Shijie Xin

Department of Vascular Surgery The First Affiliated Hospital of China Medical University No.155, Nanjingbei Street Shenyang 110001

Liaoning

China

E-mail: sjxin@cmu.edu.cn

Received for publication March 23, 2020 Accepted after revision May 30, 2020

Prepublished on Liebert Instant Online June 3, 2020 